\title{
Distance-regular graphs with a relatively small eigenvalue multiplicity
}

\author{
Jack H. Koolen \\ Department of Mathematics, POSTECH, Pohang 790-785, Republic of Korea \\ koolen@postech.ac.kr \\ Joohyung Kim* \\ Department of Mathematics Education, Wonkwang University, \\ Iksan 570-749, Republic of Korea \\ joohyung@wku.ac.kr \\ Jongyook Park \\ Department of Econometrics and O.R., Tilburg University, P.O. Box 90153, \\ 5000 LE Tilburg, The Netherlands \\ jongyook@hanmail.net
}

Submitted: Jun 4, 2012; Accepted: Dec 18, 2012; Published: Jan 7, 2013

Mathematics Subject Classifications: 05E30, 05C50

\begin{abstract}
Godsil showed that if $\Gamma$ is a distance-regular graph with diameter $D \geqslant 3$ and valency $k \geqslant 3$, and $\theta$ is an eigenvalue of $\Gamma$ with multiplicity $m \geqslant 2$, then $k \leqslant \frac{(m+2)(m-1)}{2}$.

In this paper we will give a refined statement of this result. We show that if $\Gamma$ is a distance-regular graph with diameter $D \geqslant 3$, valency $k \geqslant 2$ and an eigenvalue $\theta$ with multiplicity $m \geqslant 2$, such that $k$ is close to $\frac{(m+2)(m-1)}{2}$, then $\theta$ must be a tail. We also characterize the distance-regular graphs with diameter $D \geqslant 3$, valency $k \geqslant 3$ and an eigenvalue $\theta$ with multiplicity $m \geqslant 2$ satisfying $k=\frac{(m+2)(m-1)}{2}$.
\end{abstract}

\section{Introduction}

For definitions and preliminaries, see Sections 2 and 3. In [6], Godsil showed

\footnotetext{
${ }^{*}$ Corresponding author
} 
Theorem 1. Let $\Gamma$ be a distance-regular graph with diameter $D \geqslant 3$ and valency $k \geqslant 3$. Let $\theta$ be an eigenvalue of $\Gamma$ with multiplicity $m \geqslant 2$. Then $k \leqslant \frac{(m+2)(m-1)}{2}$.

In this paper we will give, in Theorem 13, a refined statement of this result. We show that if $\Gamma$ is a distance-regular graph with diameter $D \geqslant 3$, valency $k \geqslant 2$ and an eigenvalue $\theta$ with multiplicity $m \geqslant 2$, such that $k$ is close to $\frac{(m+2)(m-1)}{2}$, then $\theta$ must be a so-called tail. This, for example, implies that several Krein parameters vanish. Using the fact that $\theta$ is a (light) tail, we are also able to characterize in Theorem 14 the distance-regular graphs with diameter $D \geqslant 3$, valency $k \geqslant 3$ and an eigenvalue $\theta$ with multiplicity $m \geqslant 2$ satisfying $k=\frac{(m+2)(m-1)}{2}$.

In Section 2 we give the necessary definitions, and in Section 3 some preliminary results. In Section 4 we characterize the (non-bipartite) Taylor graphs as the non-bipartite distance-regular graphs with diameter at least three, having a light tail such that its accompanying eigenvalue equals -1 (Theorem 12). In Section 5 we state and prove Theorem 13 and Theorem 14.

\section{Definitions}

All the graphs considered in this paper are finite, undirected and simple (for unexplained terminology, examples and more details, see $[4,7])$. Suppose that $\Gamma$ is a connected graph with vertex set $V(\Gamma)$ and edge set $E(\Gamma)$, where $E(\Gamma)$ consists of unordered pairs of two adjacent vertices. The distance $d_{\Gamma}(x, y)$ between any two vertices $x$ and $y$ in a graph $\Gamma$ is the length of a shortest path connecting $x$ and $y$. If the graph $\Gamma$ is clear from the context, then we simply use $d(x, y)$. We define the diameter $D$ of $\Gamma$ as the maximum distance in $\Gamma$. For a vertex $x \in V(\Gamma)$, define $\Gamma_{i}(x)$ to be the set of vertices which are at distance precisely $i$ from $x(0 \leqslant i \leqslant D)$. In addition, define $\Gamma_{-1}(x)=\Gamma_{D+1}(x):=\emptyset$. We write $\Gamma(x)$ instead of $\Gamma_{1}(x)$.

A connected graph $\Gamma$ with diameter $D$ is called distance-regular if there are integers $b_{i}, c_{i}(0 \leqslant i \leqslant D)$ such that for any two vertices $x, y \in V(\Gamma)$ with $d(x, y)=i$, there are precisely $c_{i}$ neighbors of $y$ in $\Gamma_{i-1}(x)$ and $b_{i}$ neighbors of $y$ in $\Gamma_{i+1}(x)$, where we define $b_{D}=c_{0}=0$. A graph $\Gamma$ is said to be strongly regular with parameters $(v, k, \lambda, \mu)$ whenever $\Gamma$ has $v$ vertices and is regular with valency $k$, adjacent vertices of $\Gamma$ have precisely $\lambda$ common neighbors, and distinct non-adjacent vertices of $\Gamma$ have precisely $\mu$ common neighbors. Note that distance-regular graphs of diameter two are strongly regular. We define $a_{i}:=k-b_{i}-c_{i}$ for notational convenience. Note that $a_{i}=\left|\Gamma(y) \cap \Gamma_{i}(x)\right|$ holds for any two vertices $x, y$ with $d(x, y)=i(0 \leqslant i \leqslant D)$.

For a distance-regular graph $\Gamma$ and a vertex $x \in V(\Gamma)$, we denote $k_{i}:=\left|\Gamma_{i}(x)\right|$ and $p_{i j}^{h}:=\left|\left\{w \mid w \in \Gamma_{i}(x) \cap \Gamma_{j}(y)\right\}\right|$ for any $y \in \Gamma_{h}(x)$. It is easy to see that $k_{i}=\frac{b_{0} b_{1} \cdots b_{i-1}}{c_{1} c_{2} \cdots c_{i}}$ and hence it does not depend on $x$. The numbers $a_{i}, b_{i-1}$ and $c_{i}(1 \leqslant i \leqslant D)$ are called the intersection numbers, and the array $\left\{b_{0}, b_{1}, \cdots, b_{D-1} ; c_{1}, c_{2}, \cdots, c_{D}\right\}$ is called the intersection array of $\Gamma$.

Suppose that $\Gamma$ is a distance-regular graph with diameter $D \geqslant 2$ and valency $k \geqslant 2$, and let $A_{i}$ be the matrix of $\Gamma$ such that the rows and the columns of $A_{i}$ are indexed by the 
vertices of $\Gamma$ and the $(x, y)$-entry is 1 whenever $x$ and $y$ are at distance $i$ and 0 otherwise. We call $A_{i}$ the $i$ th distance matrix of $\Gamma$. We abbreviate $A:=A_{1}$ and call this the adjacency matrix of $\Gamma$. The eigenvalues of the graph $\Gamma$ are the eigenvalues of $A$.

We find that $A_{0}, A_{1}, \ldots, A_{D}$ form a basis for a commutative subalgebra $M$ of $\operatorname{Mat}_{X}(\mathbb{C})$. We call $M$ the Bose-Mesner algebra of $\Gamma$. It turns out that $A$ generates $M[1$, p. 190]. By [4, p. 45], $M$ has a second basis $E_{0}, E_{1}, \ldots, E_{D}$ of the primitive idempotents of $\Gamma$, and $A$ can be written as $A=\sum_{i=0}^{D} \theta_{i} E_{i}$, where $\theta_{i}$ is the eigenvalue of $\Gamma$ associated with $E_{i}$ $(0 \leqslant i \leqslant D)$. We denote by $m_{i}$ the multiplicity of $\theta_{i}$. For an eigenvalue $\theta=\theta_{i}$ we will also write $E_{\theta}$ instead of $E_{i}$.

For an eigenvalue $\theta$ of $\Gamma$, the sequence $\left(\omega_{i}\right)_{i=0,1, \ldots, D}=\left(\omega_{i}(\theta)\right)_{i=0,1, \ldots, D}$ satisfying $\omega_{0}=$ $\omega_{0}(\theta)=1, \omega_{1}=\omega_{1}(\theta)=\theta / k$, and

$$
c_{i} \omega_{i-1}+a_{i} \omega_{i}+b_{i} \omega_{i+1}=\theta \omega_{i} \quad(i=1,2, \ldots, D-1)
$$

is called the standard sequence corresponding to the eigenvalue $\theta$ ([4, p.128]). A sign change of $\left(\omega_{i}\right)_{i=0,1, \ldots, D}$ is a pair $(i, j)$ with $0 \leqslant i<j \leqslant D$ such that $\omega_{i} \omega_{j}<0$ and $\omega_{t}=0$ for $i<t<j$.

Let $\circ$ denote the entrywise product in $\operatorname{Mat}_{X}(\mathbb{C})$. Observe that $A_{i} \circ A_{j}=\delta_{i j} A_{i}$ for $0 \leqslant i, j \leqslant D$, so $M$ is closed under $\circ$. Thus there exist complex scalars $q_{i j}^{h}(0 \leqslant h, i, j \leqslant D)$ such that

$$
E_{i} \circ E_{j}=|V(\Gamma)|^{-1} \sum_{h=0}^{D} q_{i j}^{h} E_{h} \quad(0 \leqslant i, j \leqslant D) .
$$

By $\left[2\right.$, p. 170], $q_{i j}^{h}$ is real and nonnegative for $0 \leqslant h, i, j \leqslant D$. The $q_{i j}^{h}$ are called the Krein parameters. The graph $\Gamma$ is said to be $Q$-polynomial (with respect to the given ordering $E_{0}, E_{1}, \ldots, E_{D}$ of the primitive idempotents) whenever $q_{i j}^{h}=0$ (resp. $q_{i j}^{h} \neq 0$ ) whenever one of $h, i, j$ is greater than (resp. equal to) the sum of the other two $(0 \leqslant h, i, j \leqslant D)$ $[4$, p. 59].

For each vertex $x \in V(\Gamma)$, we let $\Delta(x)$ denote the subgraph of $\Gamma$ induced on $\Gamma(x)$. We refer to $\Delta(x)$ as the local graph at vertex $x$. We observe that $\Delta(x)$ has $k$ vertices, and is regular with valency $a_{1}$.

A graph $\Gamma$ is called bipartite if it has no odd cycle. (A distance-regular graph $\Gamma$ with diameter $D$ is bipartite if and only if $a_{1}=a_{2}=\ldots=a_{D}=0$.) An antipodal graph is a connected graph $\Gamma$ with diameter $D \geqslant 2$ for which being at distance 0 or $D$ is an equivalence relation. If, moreover, all equivalence classes have the same size $r$, then $\Gamma$ is also called an antipodal $r$-cover. A distance-regular graph $\Gamma$ with intersection array $\{k, \mu, 1 ; 1, \mu, k\}$ is called a Taylor graph. These are precisely the distance-regular antipodal 2 -covers with diameter 3 .

We define tails as follows: An eigenvalue $\theta$ of a distance-regular graph $\Gamma$ with valency $k$ is called a tail if $\theta \neq k$ and $E_{\theta} \circ E_{\theta}=\alpha J+\beta E_{\theta}+\gamma E_{\theta^{\prime}}$ for some eigenvalue $\theta^{\prime} \neq k, \theta$ and some $\alpha, \beta$ and $\gamma \neq 0$. We call $\theta^{\prime}$ the accompanying eigenvalue for the tail $\theta$. We call $\theta$ a light tail if $\beta=0$ and heavy otherwise. Note that $\alpha>0$ and $\beta \geqslant 0$. (Note that in [13], [10], they also allow $\gamma=0$ for a tail and a light tail, respectively. Note that for diameter $D \geqslant 3$ this case of $\gamma=0$ only occurs if $\Gamma$ is an antipodal distance-regular graph of diameter $D=3$ and $\theta=-1$ ([10, Theorem 4.1(b)]).) 


\section{Preliminaries}

In this section we will give some preliminary results.

The following lemma is a special case of the Absolute Bound and we state it for distance-regular graphs only.

Lemma 2. ([15]) Let $\Gamma$ be a distance-regular graph with diameter $D \geqslant 2$.

Then $\sum_{q_{i i}^{j} \neq 0} m_{j} \leqslant \frac{m_{i}\left(m_{i}+1\right)}{2}(0 \leqslant j \leqslant D)$.

The next result relates the multiplicity of an eigenvalue and its number of vertices for a strongly regular graph. A graph $\Gamma$ is called coconnected if its complement is connected.

Lemma 3. Let $\Gamma$ be a connected and coconnected strongly regular graph with $v$ vertices and distinct eigenvalues $k>\sigma>\tau$ with corresponding multiplicities $1, f, g$. Then

(i) $v \leqslant \min \left\{\frac{f(f+3)}{2}, \frac{g(g+3)}{2}\right\}$.

(ii) If $v>\frac{g(g+1)}{2}$, then $\tau$ is a light tail, that is, $\mu=\frac{-(\sigma+1) \tau\left(\tau+\sigma^{2}\right)}{\tau-\sigma(\sigma+2)}$.

(iii) If $v>\frac{f(f+1)}{2}$, then $\sigma$ is a light tail, that is, $\mu=\frac{-(\tau+1) \sigma\left(\sigma+\tau^{2}\right)}{\sigma-\tau(\tau+2)}$.

(iv) If $v=\frac{g(g+3)}{2}$, then

$$
\begin{aligned}
\mu & =\sigma^{3}(2 \sigma+3), \\
k & =2 \mu, \\
\lambda & =\sigma\left(2 \sigma^{3}+\sigma^{2}-3 \sigma+1\right), \\
v & =(2 \sigma+1)^{2}\left(2 \sigma^{2}+2 \sigma-1\right), \\
\tau & =-\sigma^{2}(2 \sigma+3),
\end{aligned}
$$

and $\sigma>0$ and $\tau<-1$ are integers except for the case $\sigma=\frac{-1+\sqrt{5}}{2}$, $\tau=\frac{-1-\sqrt{5}}{2}$ and $\Gamma$ is the pentagon.

(v) If $v=\frac{f(f+3)}{2}$, then

$$
\begin{aligned}
\mu & =\tau^{3}(2 \tau+3) \\
k & =2 \mu \\
\lambda & =\tau\left(2 \tau^{3}+\tau^{2}-3 \tau+1\right) \\
v & =(2 \tau+1)^{2}\left(2 \tau^{2}+2 \tau-1\right), \\
\sigma & =-\tau^{2}(2 \tau+3)
\end{aligned}
$$

and $\sigma>0$ and $\tau<-1$ are integers except for the case $\sigma=\frac{-1+\sqrt{5}}{2}$, $\tau=\frac{-1-\sqrt{5}}{2}$ and $\Gamma$ is the pentagon.

Proof: (i) This follows from the absolute bound, Lemma 2. See also [19, p.169]. (ii) It follows from [15, Theorem 2] and [5, Theorem 6.1]. 
(iii) If we take the complement of $\Gamma$ then it is a strongly regular graph satisfying (ii), and the result follows easily.

(iv) See [14] (cf. [19, p.169-170]).

(v) If we take the complement of $\Gamma$ then it is a strongly regular graph satisfying (iv), and the result follows easily.

Next, we introduce the Fundamental Bound and tight distance-regular graphs.

Lemma 4. ([9, Theorem 6.2]) Let $\Gamma$ be a distance-regular graph with diameter $D \geqslant 3$, valency $k$ and distinct eigenvalues $k=\theta_{0}>\theta_{1}>\ldots>\theta_{D}$. Then the following inequality holds.

$$
\left(\theta_{1}+\frac{k}{a_{1}+1}\right)\left(\theta_{D}+\frac{k}{a_{1}+1}\right) \geqslant-\frac{k a_{1} b_{1}}{\left(a_{1}+1\right)^{2}}
$$

We refer to (1) as the Fundamental Bound. A distance-regular graph $\Gamma$ is tight if $\Gamma$ is not bipartite and equality holds in (1).

The next lemma gives some known results on tight distance-regular graphs.

Lemma 5. Let $\Gamma$ be a distance-regular graph with diameter $D \geqslant 3$, valency $k$ and distinct eigenvalues $k=\theta_{0}>\theta_{1}>\ldots>\theta_{D}$. Then

(i) $([9$, Theorem 12.6]) $\Gamma$ is tight if and only if for all $x \in V(\Gamma)$, the local graph $\Delta(x)$ is connected strongly regular with distinct eigenvalues $a_{1},-1-\frac{b_{1}}{\theta_{D}+1},-1-\frac{b_{1}}{\theta_{1}+1}$.

(ii) $\left(\left[9\right.\right.$, Theorem 11.7]) If $\Gamma$ is tight, then the intersection number $a_{D}$ satisfies $a_{D}=0$.

(iii) $\left(\left[18\right.\right.$, Lemma 3.5], cf.[17]) If $\Gamma$ is tight, then the Krein parameter $q_{1 D}^{i}$ satisfies $q_{1 D}^{i}=0$ unless $i=D-1(0 \leqslant i \leqslant D)$.

The next result is due to Terwilliger and concerns the eigenvalues of the local graph $\Delta(x)$ at a vertex $x$ of a distance-regular graph $\Gamma$.

Proposition 6. ([4, Theorem 4.4.4]) Let $\Gamma$ be a distance-regular graph with diameter $D \geqslant 3$, valency $k$ and distinct eigenvalues $k=\theta_{0}>\theta_{1}>\ldots>\theta_{D}$ with corresponding multiplicities $1=m_{0}, m_{1}, \ldots, m_{D}$. If $\theta_{i}$ has multiplicity $m_{i}$ with $1<m_{i}<k$, then $\theta_{i} \in\left\{\theta_{1}, \theta_{D}\right\}$. Putting $b=\frac{b_{1}}{\theta_{i}+1}$ we have that each local graph $\Delta(x)$ has eigenvalue $-1-b$ with multiplicity at least $k-m_{i}$; in case $-1-b=a_{1}$ its multiplicity is at least $k-m_{i}+1$.

The following lemma is a consequence of Proposition 6.

Lemma 7. Let $\Gamma$ be a distance-regular graph with diameter $D \geqslant 3$, valency $k$ and distinct eigenvalues $k=\theta_{0}>\theta_{1}>\ldots>\theta_{D}$ with corresponding multiplicities $1=m_{0}, m_{1}, \ldots, m_{D}$. Then $m_{1}+m_{D} \geqslant k+1$.

Proof: As the sum of the multiplicities of $-1-\frac{b_{1}}{\theta_{1}+1}$ and $-1-\frac{b_{1}}{\theta_{D}+1}$ as eigenvalues of the local graph at vertex $x$ is at most $k-1$ if $-1-\frac{b_{1}}{\theta_{D}+1} \neq a_{1}$ and at most $k$ if equals $a_{1}$, the result follows.

In the next lemma we show that the accompanying eigenvalue of a light tail $\theta$ is the third-largest eigenvalue, if $\theta$ is the second-largest eigenvalue. 
Lemma 8. Let $\Gamma$ be a distance-regular graph with diameter $D \geqslant 3$, valency $k$ and distinct eigenvalues $k=\theta_{0}>\theta_{1}>\ldots>\theta_{D}$. If $\theta=\theta_{1}$ is a light tail, then the accompanying eigenvalue $\theta^{\prime}$ satisfies $\theta^{\prime}=\theta_{2}$.

Proof: Let $E_{i}$ be the primitive idempotent corresponding to $\theta_{i}$. Now $E_{1} \circ E_{1}=\alpha E_{0}+\beta E_{i}$, where $\alpha$ and $\beta$ are positive numbers. As the standard sequence corresponding to $\theta_{1}$ is strictly decreasing, this implies that the standard sequence corresponding to $\theta_{i}$ has at most two sign changes ([10, Theorem 4.1(iii)]). But as $i \neq 0,1$ it follows that $i=2$.

\section{Characterizations of Taylor graphs}

In this section we will give some characterizations of the Taylor graphs. We start with the following result, due to Taylor.

Lemma 9. ([4, Proposition 1.5.1, Theorem 1.5.3])

(i) If $\Gamma$ is a Taylor graph with valency $k$, then for every $x \in V(\Gamma)$, the local graph $\Delta(x)$ is strongly regular with parameters $\left(v^{\prime}, k^{\prime}, \lambda^{\prime}, \mu^{\prime}\right)$ and satisfies $a_{1}=k^{\prime}=2 \mu^{\prime}$, and $v^{\prime}=k$.

(ii) If $\Delta$ is a (non-complete) connected strongly regular graph with $\left(v^{\prime}, k^{\prime}, \lambda^{\prime}, \mu^{\prime}\right)$ such that $k^{\prime}=2 \mu^{\prime}$, then there exists a Taylor graph $\Gamma$ and a vertex $x$ of $\Gamma$ such that the local graph $\Delta(x)$ of $\Gamma$ is isomorphic to $\Delta$.

Remark: We denote by Tay $(\Delta)$, the Taylor graph as in Lemma $9(i i)$, where $\Delta$ is a (noncomplete) connected strongly regular graph with $\left(v^{\prime}, k^{\prime}, \lambda^{\prime}, \mu^{\prime}\right)$ satisfying $k^{\prime}=2 \mu^{\prime}$.

The next result gives some sufficient conditions for a distance-regular graph to be tight.

Lemma 10. Let $\Gamma$ be a distance-regular graph with diameter $D \geqslant 3$, valency $k$ and distinct eigenvalues $k=\theta_{0}>\theta_{1}>\ldots>\theta_{D}$ with corresponding multiplicities $1=m_{0}, m_{1}, \ldots, m_{D}$. Then the following hold.

(i) If $m_{1}+m_{D}=k+1$, then $\Gamma$ is an antipodal 2-cover, and $\Gamma$ is tight or bipartite.

(ii) If for all vertices $x$ the local graph $\Delta(x)$ is strongly regular and $m_{1}, m_{D}<k$, then $\Gamma$ is tight.

Proof: (i) If $m_{1}+m_{D}=k+1$, then we need to consider two cases: $m_{D}=1$ and $m_{D} \geqslant 2$. If $m_{D}=1$, then $\Gamma$ is bipartite and $\theta_{D}=-k$ by [4, Proposition 4.4.8(i)]. If $m_{i}=1$ and $i \geqslant 1$, then $i=D, \theta_{D}=-k$ and $\Gamma$ is bipartite. So from now we may assume $m_{1} \geqslant 2$ and $m_{D} \geqslant 2$. Now let $m_{D} \geqslant 2$. Then $m_{1}=k+1-m_{D}<k$. If $\theta_{D}=-1-\frac{b_{1}}{\theta_{1}+1}$, then the local graph $\Delta(x)$ at vertex $x$ has eigenvalues $a_{1}$ and $-1-\frac{b_{1}}{\theta_{1}+1}$ with corresponding multiplicities $k-m_{D}+1$ and $k-m_{1}$ by Proposition 6. So this means that $\Delta(x)$ is a disjoint union of cliques. Since $\theta_{1}>0$, we find that $-1-\frac{b_{1}}{\theta_{1}+1}<-1$. But it is not possible. So we find that $\theta_{D} \neq-1-\frac{b_{1}}{\theta_{1}+1}$. Then again by Proposition 6 we find that for all vertices $x$ the local graph $\Delta(x)$ has eigenvalues $a_{1},-1-\frac{b_{1}}{\theta_{D}+1},-1-\frac{b_{1}}{\theta_{1}+1}$ with corresponding multiplicities 1 , 
$k-m_{D}, k-m_{1}$. So this means that $\Delta(x)$ is strongly regular by [7, Lemma 10.1.5], and hence by Lemma $5(\mathrm{i})$, we find $\Gamma$ is tight. So we have shown that $\Gamma$ is tight or bipartite. This means that $a_{D}=0$ by Lemma 5(ii). By [8] it follows that $k_{D}=1$ as otherwise $-1-\frac{b_{1}}{\theta_{1}+1}$ has multiplicity at least $k+1-m_{1}$ in $\Delta(x)$ for any vertex $x$. This shows (i). (ii) Let $x$ be a vertex of $\Gamma$ and consider the local graph $\Delta(x)$. Proposition 6 implies that $-1-\frac{b_{1}}{\theta_{1}+1}$ and $-1-\frac{b_{1}}{\theta_{D}+1}$ are both eigenvalues of $\Delta(x)$. Now $-1-\frac{b_{1}}{\theta_{1}+1} \neq-1$, so that means $\Delta(x)$ is not the disjoint union of cliques, and hence is connected. But this shows that $\Gamma$ is tight in similar fashion as in (i).

Remark: (i) The bipartite distance-regular graphs with an eigenvalue having multiplicity $k$ are determined by N. Yamazaki [21] and K. Nomura [16]. They found the following:

(a) $2 d$-gons,

(b) complete bipartite graphs,

(c) complements of $2 \times(k+1)$-grids,

(d) Hadamard graphs,

(e) antipodal 2-covers with the intersection array $\{k, k-1, k-c, c, 1 ; 1, c$, $k-c, k-1, k\}$, where $k=\gamma\left(\gamma^{2}+3 \gamma+1\right), c=\gamma(\gamma+1)$ and $\gamma \geqslant 2$,

(f) hypercubes.

For the fifth case, if $\gamma=2$, then the graph is 2-cover of Higman-Sims graph, and for $\gamma \geqslant 3$, no graph is known.

(ii) The Taylor graphs have $m_{1}+m_{3}=k+1$. Besides them there are feasible intersection arrays known for diameter 4 with $m_{1}+m_{4}=k+1$. These are

$$
\begin{aligned}
& \{56,45,12,1 ; 1,12,45,56\}, \\
& \{115,96,20,1 ; 1,20,96,115\}, \\
& \{204,175,30,1 ; 1,30,175,204\} \text { and, } \\
& \{329,288,42,1 ; 1,42,288,329\} .
\end{aligned}
$$

For the first intersection array, it is known that there are no distance-regular graphs with this intersection array $([3,11.4 .6$ Theorem $])$. There are no feasible intersection arrays known for larger diameter.

In Theorem 12 below, we show that the (non-bipartite) Taylor graphs are the distanceregular graphs with diameter $D \geqslant 3$, valency $k$ and intersection number $a_{1} \neq 0$ having a light tail such that its accompanying eigenvalue equals -1 . To show this result we first need the following lemma.

Lemma 11. Let $\Gamma$ be a distance-regular graph with diameter $D \geqslant 3$, valency $k$, intersection number $a_{1} \neq 0$ and distinct eigenvalues $k=\theta_{0}>\theta_{1}>\ldots>\theta_{D}$. Let $\theta$ be a light tail of $\Gamma$ with standard sequence $1=\omega_{0}, \omega_{1}, \ldots, \omega_{D}$ and let $\theta^{\prime}$ be the accompanying eigenvalue of $\theta$. For all $x \in V(\Gamma)$, let the local graph $\Delta(x)$ be a (non-complete) strongly regular graph with parameters $\left(v^{\prime}=k, k^{\prime}=a_{1}, \lambda^{\prime}, \mu^{\prime}\right)$. Then the following statements are equivalent. 
(i) $\theta^{\prime}=-1$.

(ii) $\theta$ is a root of $x^{2}-\left(a_{1}-b_{1}\right) x-k$.

(iii) $k^{\prime}=2 \mu^{\prime}$.

(iv) $\omega_{2}=-\omega_{1}$.

Proof: The equivalence (i) $\Leftrightarrow($ ii) follows from [10, Theorem 4.1(a)].

The equivalence (ii) $\Leftrightarrow$ (iii) follows from [10, Corollary 6.3].

The equivalence (ii) $\Leftrightarrow$ (iv) is straightforward.

In the next result we show that any of the 4 statements in Lemma 11 is equivalent with $\Gamma$ be a Taylor graph.

Theorem 12. Let $\Gamma$ be a distance-regular graph with diameter $D \geqslant 3$, valency $k$, intersection number $a_{1} \neq 0$ and distinct eigenvalues $k=\theta_{0}>\theta_{1}>\ldots>\theta_{D}$. Let $\theta \neq \pm k$ be an eigenvalue of $\Gamma$. Then the following statements are equivalent:

(i) $\theta$ is a light tail of $\Gamma$ with standard sequence $1=\omega_{0}, \omega_{1}, \ldots, \omega_{D}$ such that its accompanying eigenvalue $\theta^{\prime}$ equals -1 .

(ii) $\Gamma$ is a Taylor graph and $\theta \in\left\{\theta_{1}, \theta_{3}\right\}$.

Proof: (i) $\Rightarrow$ (ii) As $a_{1} \neq 0$ and $\theta$ is a light tail it follows that $\theta \in\left\{\theta_{1}, \theta_{D}\right\}$ by $[10$, Remarks 3.3(iii)]. If $\theta=\theta_{1}$, then $\theta_{2}=\theta^{\prime}=-1$ by Lemma 8. If $D \geqslant 4$, then $\theta_{2} \geqslant \min \left\{0, a_{2}, a_{4}\right\} \geqslant 0$. This implies that $D=3$. By [10, Theorem 5.1] and Lemma 11 we find $c_{3}=k \frac{\omega_{3}\left(1-\omega_{1}\right)}{\omega_{3}-\omega_{2}}=k \frac{\omega_{3}\left(1-\omega_{1}\right)}{\omega_{3}+\omega_{1}}$. This implies $c_{3}=k$ and $\omega_{3}=-1$ as $\omega_{1}>0$ and hence $\Gamma$ is an antipodal $r$-cover. By [4, p.142-143], $\omega_{3}=-1 /(r-1)$ and hence $\Gamma$ is a Taylor graph. Let us assume that $\theta=\theta_{D}$, then we need to consider two cases: $D=3$ and $D \geqslant 4$. If $D=3$, then let $\alpha$ be the largest root of $x^{2}-\left(a_{1}-b_{1}\right) x-k$. Let Tay $(\Delta)$ be the Taylor graph corresponding to $\Delta=\Delta(x)$ as in Lemma 9(ii). Here note that as $\theta$ is a light tail the local graph $\Delta=\Delta(x)$ is a (non-complete) strongly regular graph with parameters $\left(v^{\prime}=k, k^{\prime}=a_{1}, \lambda^{\prime}, \mu^{\prime}\right)$ and it satisfies $k^{\prime}=2 \mu^{\prime}$ by Lemma 11 . Now $\Delta$ has the smallest eigenvalue $-1-\frac{b_{1}}{\alpha+1}$ as $\alpha$ is an eigenvalue of $\operatorname{Tay}(\Delta)$ and $\operatorname{Tay}(\Delta)$ is tight. This implies $\theta_{1} \leqslant \alpha\left(\left[4\right.\right.$, Theorem 4.4.3]). But then $a_{1}+a_{2}+a_{3}=k+\theta_{1}+\theta_{2}+\theta_{3} \leqslant k+\alpha+\theta_{2}+\theta_{3}=2 a_{1}$ as $\operatorname{Tay}(\Delta)$ has eigenvalues $k, \alpha, \theta_{2}, \theta_{3}$. Hence $a_{1} \geqslant a_{2}+a_{3}$. But $a_{2}+a_{3} \geqslant a_{1}$ by $[11$, Proposition 4]. So $a_{2}+a_{3}=a_{1}$ and this implies $a_{3}=0$ and $b_{2}=1$ and hence $\Gamma$ is a Taylor graph. If $D \geqslant 4$, then by [12, Theorem 3.1(iii)], $\theta_{1} \geqslant \frac{a_{1}+\sqrt{a_{1}^{2}+4 k}}{2}>a_{1}+1$. But again from the proof of $D=3$ we have $\theta_{1} \leqslant \alpha$, where $\alpha$ is the largest root of $x^{2}-\left(a_{1}-b_{1}\right) x-k$. But if we evaluate the polynomial $x^{2}-\left(a_{1}-b_{1}\right) x-k$ in point $a_{1}+1$ we see that it is always non-negative. This means that $\alpha \leqslant a_{1}+1$ and $\alpha \geqslant \theta_{1}>a_{1}+1$, a contradiction. So this case can not occur.

(ii) $\Rightarrow$ (i) It is easily checked that if $\Gamma$ is a Taylor graph then $\theta \in\left\{\theta_{1}, \theta_{3}\right\}$ is a light tail and its accompanying eigenvalue $\theta^{\prime}$ equals -1 . 


\section{The refined bound}

In this section, we will show the following refined version of Theorem 1.

Theorem 13. Let $\Gamma$ be a distance-regular graph with diameter $D \geqslant 3$, valency $k \geqslant 2$ and distinct eigenvalues $k=\theta_{0}>\theta_{1}>\ldots, \theta_{D}$. Let $\theta \neq \pm k$ be an eigenvalue of $\Gamma$ with multiplicity $m \geqslant 2$. Then $k \leqslant \frac{(m+2)(m-1)}{2}$. More precisely, the following hold.

(i) If $m=2$, then $k=2$.

(ii) If $\theta$ is not a tail and $m \geqslant 3$, then $k \leqslant \frac{(m-1)(m+4)}{4}$.

(iii) If $\theta$ is a heavy tail with $\theta^{\prime} \notin\left\{\theta_{1}, \theta_{D}\right\}$ and $m \geqslant 3$, then $k \leqslant \frac{(m+1)(m-2)}{2}$.

(iv) If $\theta$ is a heavy tail with $\theta^{\prime} \in\left\{\theta_{1}, \theta_{D}\right\}$ and $m \geqslant 3$, then $k \leqslant \frac{(m-2)(m+3)}{2}$.

(v) If $\theta$ is a light tail, then $k \leqslant \frac{(m+2)(m-1)}{2}$.

Proof: Let $\theta=\theta_{i} \neq \pm k$ be an eigenvalue of $\Gamma$ with multiplicity $m=m_{i}$. [4, Proposition 4.4.8(ii)] shows $k=2$ if and only if $m=2$. This shows (i). So from now on we may assume $m \geqslant 3$ and $k \geqslant 3$. We will first consider the case $m<k$ and later we will consider $m \geqslant k$. Let us first assume $m<k$. Then $i \in\{1, D\}$ by Proposition 6 , and $a_{1} \neq 0$ by [10, Theorem 3.2]. If there are at least two distinct $j_{1}, j_{2} \notin\{0, i\}$ satisfying $q_{i i}^{j_{1}} \neq 0 \neq q_{i i}^{j_{2}}$, then by Lemma 2 and Lemma 7 we have $\frac{m(m+1)}{2} \geqslant m_{0}+m_{j_{1}}+m_{j_{2}} \geqslant 1+k+k-m+1$ and hence $k \leqslant \frac{(m-1)(m+4)}{4}$. If $q_{i i}^{j}=0$ for all $j \notin\{0, i\}$, then by [10, Theorem 4.1(b)], $\Gamma$ is antipodal with diameter 3 and $\theta=\theta_{2}=-1$. But then $m=k$. This shows (ii) if $m<k$. Now let us assume $\theta$ is a tail and $\theta^{\prime}$ its accompanying eigenvalue. Let $m^{\prime}$ be the multiplicity of $\theta^{\prime}$. If $\theta$ is a heavy tail with $\theta^{\prime} \notin\left\{\theta_{1}, \theta_{D}\right\}$, then by Lemma 2 and Proposition $6, \frac{m(m+1)}{2} \geqslant 1+m+k$ and this shows (iii) if $m<k$. If $\theta$ is a heavy tail with $\theta^{\prime} \in\left\{\theta_{1}, \theta_{D}\right\}$, then by Lemma 2, Proposition 6, and Lemma 7, $\frac{m(m+1)}{2} \geqslant 1+m+m^{\prime} \geqslant 1+m+k+1-m=k+2$. But if $\frac{m(m+1)}{2}=k+2$, then $m+m^{\prime}=k+1$ and it follows by Lemma 10 that $\Gamma$ is tight. But $q_{1 D}^{j}=0$ if $j \neq D-1$ by Lemma 5 (iii), so this give a contradiction. This shows (iv) when $m<k$. Now if $\theta$ is a light tail, then for all vertices $x$ the local graph $\Delta(x)$ is strongly regular by [10, Corollary 6.3]. If $m^{\prime}<k$, then $\left\{\theta, \theta^{\prime}\right\}=\left\{\theta_{1}, \theta_{D}\right\}$ and by Lemma 10 (ii) $\Gamma$ is tight. But this is not possible by Lemma 5(iii). This means $m^{\prime} \geqslant k$. Now by Lemma $2, \frac{m(m+1)}{2} \geqslant m_{0}+m^{\prime} \geqslant 1+k$. This shows (v). So we have shown the theorem if $m<k$. As $m \leqslant \frac{(m-1)(m+4)}{4}, m \leqslant \frac{(m-2)(m+3)}{2}$, and $m \leqslant \frac{(m+2)(m-1)}{2}$ if $m \geqslant 3$, it follows that cases (ii), (iv), and (v) also hold if $m \geqslant k \geqslant 3$. For case (iii) and $m \geqslant k \geqslant 3$ we see that $m \leqslant \frac{(m+1)(m-2)}{2}$ unless $m=3$. If $m=3$ and $m \geqslant k \geqslant 3$, then we see that $k=3$ and $a_{1}=0$ as $D \geqslant 3$. But then $\theta$ is a light tail, a contradiction with the assumption that $\theta$ is a heavy tail.

In the following theorem, we characterize the distance-regular graphs with valency at least three which attain the bound in Theorem 13. 
Theorem 14. Let $\Gamma$ be a distance-regular graph with diameter $D \geqslant 3$, valency $k \geqslant 3$ and an eigenvalue $\theta$ having multiplicity $m \geqslant 2$. Then the following statements are equivalent. (i) $k=\frac{(m+2)(m-1)}{2}$.

(ii) $\Gamma$ is a Taylor graph with intersection array $\left\{(2 \alpha+1)^{2}\left(2 \alpha^{2}+2 \alpha-1\right), 2 \alpha^{3}(2 \alpha+\right.$ $\left.3), 1 ; 1,2 \alpha^{3}(2 \alpha+3),(2 \alpha+1)^{2}\left(2 \alpha^{2}+2 \alpha-1\right)\right\}$ where $\alpha$ is an integer $\neq 0,-1$ or $\alpha=\frac{-1 \pm \sqrt{5}}{2}$, (and $m=4 \alpha^{2}+4 \alpha-1$ ).

Proof: (i) $\Rightarrow$ (ii) The only distance-regular graphs with an eigenvalue having multiplicity 2 are the polygons. So $\theta$ has multiplicity $m \geqslant 3$. As $m<\frac{(m+2)(m-1)}{2}$ if $m \geqslant 3$, we have $m<k$ and hence $a_{1} \neq 0$. By Theorem 13, the eigenvalue $\theta$ is a light tail. To complete the proof, we will show that for any vertex $x$ of $\Gamma$, the local graph $\Delta(x)$ at the vertex $x$ is a strongly regular graph with parameters $\left(v^{\prime}, k^{\prime}, \lambda^{\prime}, \mu^{\prime}\right)$ satisfying $k^{\prime}=2 \mu^{\prime}$. Then by Lemma 11 the accompanying eigenvalue $\theta^{\prime}$ of $\theta$ is equal to -1 , and hence by Theorem 12 the graph $\Gamma$ is a Taylor graph with the parameters as stated in the theorem.

Let $x$ be a vertex of $\Gamma$. Then the local graph $\Delta(x)$ is a strongly regular graph. If $\Delta(x)$ is not connected, then $\Delta(x)$ is the disjoint union of $\frac{k}{a_{1}+1}$ complete graphs with $a_{1}+1$ vertices. Then by [10, Corollary 6.3], we have

$$
\theta=\theta_{D}=-1-\frac{b_{1}}{a_{1}+1}=\frac{-k}{a_{1}+1}
$$

and also by [10, Theorem 3.2] we have

$$
m=k-\frac{b_{1}}{a_{1}+1} \geqslant \frac{k}{2}+1 .
$$

As $k=\frac{(m+2)(m-1)}{2} \geqslant 2 m-1$ if $m \geqslant 3$, we find that $\Delta(x)$ must be connected. By [10, Corollary 6.3] we find that $\Delta(x)$ has an eigenvalue $\frac{a_{1} \theta}{\theta+k}$ with multiplicity $m-1$. Now by parts (iv) and (v) in Lemma 3, we find that the local graph $\Delta(x)$ at the vertex $x$ is strongly regular with parameters $\left(v^{\prime}, k^{\prime}, \lambda^{\prime}, \mu^{\prime}\right)=\left((2 \alpha+1)^{2}\left(2 \alpha^{2}+2 \alpha-1\right), 2 \alpha^{3}(2 \alpha+\right.$ 3), $\left.\alpha\left(2 \alpha^{3}+\alpha^{2}-3 \alpha+1\right), \alpha^{3}(2 \alpha+3)\right)$ satisfying $k^{\prime}=2 \mu^{\prime}$, where $\alpha$ is an integer $\neq 0,-1$ or $\alpha=\frac{-1 \pm \sqrt{5}}{2}$. This shows (i).

(ii) $\Rightarrow$ (i) Trivial.

This finishes the proof.

Remark: Note that the distance-2 graph of a graph $\Gamma=(V(\Gamma), E(\Gamma))$ has as vertex set $V(\Gamma)$ and two vertices are adjacent if they have distance 2 in $\Gamma$. Then the distance-2 graph of a Taylor graph with intersection array

$$
\left\{(2 \alpha+1)^{2}\left(2 \alpha^{2}+2 \alpha-1\right), 2 \alpha^{3}(2 \alpha+3), 1 ; 1,2 \alpha^{3}(2 \alpha+3),(2 \alpha+1)^{2}\left(2 \alpha^{2}+2 \alpha-1\right)\right\},
$$

where $\alpha$ is an integer $\neq 0,1$ or $\alpha=\frac{-1 \pm \sqrt{5}}{2}$, is again a Taylor graph with intersection array

$$
\left\{(2 \beta+1)^{2}\left(2 \beta^{2}+2 \beta-1\right), 2 \beta^{3}(2 \beta+3), 1 ; 1,2 \beta^{3}(2 \beta+3),(2 \beta+1)^{2}\left(2 \beta^{2}+2 \beta-1\right)\right\},
$$

where $\beta=-\alpha-1$. 
Also, the following hold:

(i) $\Gamma$ is the Icosahedron if $\alpha=\frac{-1 \pm \sqrt{5}}{2}$,

(ii) $\Gamma$ is the Gosset graph if $\alpha=1$,

(iii) $\Gamma$ is the distance-2 graph of Gosset graph if $\alpha=-2$,

(iv) $\Gamma$ is the Tay(McLaughlin graph) (see [20]) if $\alpha=-3$,

(v) $\Gamma$ is the distance-2 graph of Tay(McLaughlin graph) if $\alpha=2$,

(vi) For the other $\alpha$ nothing is known.

\section{Acknowledgements}

We would like to thank Edwin van Dam for his careful reading and comments. The third author is (partly) financed by the mathematics cluster DIAMANT of the Netherlands Organisation for Scientific Research (NWO).

\section{References}

[1] E. Bannai and T. Ito, Algebraic Combinatorics I: Association Schemes, Benjamin/Cummings, London, 1984.

[2] N. Biggs, Algebraic Graph Theory, Second edition, Cambridge University Press, Cambridge, 1993.

[3] A.E. Brouwer, Corrections and additions to the book 'Distance-regular Graphs', http://www.win.tue.nl/ aeb/drg/BCN-ac.ps.gz.

[4] A. E. Brouwer, A. M. Cohen and A. Neumaier, Distance-regular graphs, SpringerVerlag, Berlin, 1989.

[5] P. J. Cameron, J.-M. Goethals, J. J. Seidel, Strongly regular graphs having strongly regular subconstituents, J. Algebra 55 (1978), 257-280.

[6] C. D. Godsil, Bounding the diameter of distance-regular graphs, Combinatorica 8 (1988), 333-343.

[7] C. D. Godsil, Algebraic Combinatorics, Chapman and Hall Inc., New York, 1993.

[8] C. D. Godsil, J. H. Koolen, On the multiplicity of eigenvalues of distance-regular graphs, Linear Algebra Appl. 226-228 (1995), 273-275.

[9] A. Jurišić, J. H. Koolen, P. Terwilliger, Tight distance-regular graphs, J. Algebraic Combin. 12 (2000), 163-197.

[10] A. Jurišić, P. Terwilliger, A. Žitnik, Distance-regular graphs with light tails, European J. Combin. 31 (2010), 1539-1552.

[11] J. H. Koolen, J. Park, Distance-regular graphs with $a_{1}$ or $c_{2}$ at least half the valency, J. Combin. Theory Ser. A 119 (2012), 546-555.

[12] J. H. Koolen, J. Park, H. Yu, An inequality involving the second largest and smallest eigenvalue of a distance-regular graph, Linear Algebra Appl. 434 (2011), 2404-2412. 
[13] M. S. Lang, Tails of bipartite distance-regular graphs, European J. Combin. 23 (2002), 1015-1023.

[14] A. Neumaier, Strongly regular graphs with smallest eigenvalue $-m$, Arch. Math. (Basel) 33 (1979/80), 392-400.

[15] A. Neumaier, New inequalities for the parameters of an association scheme, Combinatorics and graph theory (Calcutta, 1980), 365-367.

[16] K. Nomura, Spin models on bipartite distance-regular graphs, J. Combin. Theory Ser. B 64 (1995), 300-313.

[17] A. A. Pascasio, Tight graphs and their primitive idempotents, J. Algebraic Combin. 10 (1999), 47-59.

[18] A. A. Pascasio, Tight distance-regular graphs and the $Q$-polynomial property, Graphs Comb., 17 (2001), 149-169.

[19] J. J. Seidel, Strongly regular graphs, in: Surveys in Combinatorics, Proc. 7th Brit. Comb. Conf., B. Bollobás (ed.), London Math. Soc. Lecture Note Series 38, Cambridge 1979, 157-180.

[20] D.E. Taylor, Regular 2-graphs, Proc. London Math. Soc. (3) 35 (1977), 257-274.

[21] N. Yamazaki, Bipartite distance-regular graphs with an eigenvalue of multiplicity $k$, J. Combin. Theory Ser. B 66 (1996), 34-37. 